University of Texas Rio Grande Valley

ScholarWorks @ UTRGV

Psychological Science Faculty Publications and

Presentations

$6-2022$

\title{
One more wall to cross: The role of psychologists in addressing integrated health among undocumented Latinx immigrants in inpatient medical settings
}

\author{
Luz M. Garcini \\ University of Texas Health Science - San Antonio \\ Amanda Venta \\ Alfonso Mercado \\ The University of Texas Rio Grande Valley \\ Thania Galvan \\ Casandra L. Compean
}

See next page for additional authors

Follow this and additional works at: https://scholarworks.utrgv.edu/psy_fac

Part of the Psychology Commons

\section{Recommended Citation}

Garcini, L. M., Venta, A., Mercado, A., Galvan, T., Compean, C. L., Guerrero, G., \& Domenech Rodríguez, M. M. (2022). One more wall to cross: The role of psychologists in addressing integrated health among undocumented Latinx immigrants in inpatient medical settings. Psychotherapy. Advance online publication. https://doi.org/10.1037/pst0000381

This Article is brought to you for free and open access by the College of Liberal Arts at ScholarWorks @ UTRGV. It has been accepted for inclusion in Psychological Science Faculty Publications and Presentations by an authorized administrator of ScholarWorks @ UTRGV. For more information, please contact justin.white@utrgv.edu, william.flores01@utrgv.edu. 


\section{Authors}

Luz M. Garcini, Amanda Venta, Alfonso Mercado, Thania Galvan, Casandra L. Compean, Gabriela Guerrero, and Melanie M. Domenech Rodriguez 


\title{
One More Wall to Cross: The Role of Psychologists in Addressing Integrated Health Among Undocumented Latinx Immigrants in Inpatient Medical Settings
}

\author{
Luz M. Garcini ${ }^{1}$, Amanda Venta ${ }^{2}$, Alfonso Mercado ${ }^{3}$, Thania Galvan ${ }^{4,5}$, Casandra L. $^{2}$ \\ Compean $^{1}$, Gabriela Guerrero ${ }^{1}$, Melanie M. Domenech Rodríguez ${ }^{6}$ \\ ${ }^{1}$ University of Texas Health Science Center San Antonio, Joe R. and Teresa Lozano Long School \\ of Medicine, Department of Medicine \\ 2 University of Houston, Department of Psychology \\ ${ }^{3}$ University of Texas Rio Grande Valley, Department of Psychological Science \\ 4 University of Denver, Department of Psychology \\ ${ }^{5}$ University of Illinois, Institute for Juvenile Research \\ ${ }^{6}$ Utah State University, Department of Psychology
}

\section{Abstract}

Over the past decade, there has been increased attention to the benefits of an integrated healthcare care model in which psychologists play a vital role collaborating with other health providers to deliver services in inpatient medical settings, such as emergency departments and acute care medical units. Psychologists working in inpatient medical settings can facilitate access to mental health services and referrals, reduce mental health stigma, improve health outcomes associated with insight into psychosocial factors that affect health and illness, improve communication between patients and providers, and increase sensitivity in addressing the health needs of diverse patients. Psychological services in inpatient medical units can be particularly valuable for marginalized populations, such as undocumented Latinx immigrants, who may not otherwise have access to services that can address the compounded effect that psychosocial stressors may have on their physical health. We illustrate the critical role that psychologists can play in inpatient medical settings to address the complex health needs of Latinx immigrants. In this case study, an undocumented young woman presented to an emergency department with acute liver failure. We focus on psychologists' specific contributions as part of the integrated medical team and how it may have impacted treatment effectiveness. We also provide practical assessment and treatment recommendations, along with proposing advocacy efforts to facilitate access to culture and context sensitive treatments for undocumented immigrants with complex health issues.

Correspondence: Luz M. Garcini, University of Texas Health Sciences Center San Antonio, Joe R. and Teresa Lozano Long School of Medicine, 7703 Floyd Curl Drive, San Antonio, Texas, 78229., garcini@uthscsa.edu.

Authors contributions: All authors contributed to the study conceptualization. Material preparation was performed by Luz Garcini,

Thania Galvan, Casandra Compean and Gabriela Guerrero. The first draft of the manuscript was written by Luz Garcini, Amanda

Venta, Alfonso Mercado, and Melanie M. Domenech Rodríguez. All authors commented and edited on previous versions of the

manuscript. All authors read and approved the final manuscript.

Conflict of Interest: We have no conflicts of interest to disclose. 


\section{Keywords}

Undocumented; immigrants; mental health; physical health; integrated healthcare

Undocumented immigrants and individuals living in "mixed-status" families, that is, families in which at least one member has an undocumented immigration legal status, make up a considerable proportion of the United States (U.S.) population. A study using advanced demographic modeling proposed that the number of undocumented immigrants residing in the U.S. nearly doubles that of previous estimates, placing the current U.S. undocumented population at 22.1 million (Fazel-Zarandi et al., 2018). As undocumented immigrants establish their families in the U.S., they become settled and less likely to return to their countries of origin (Passel et al., 2014). Unfortunately, the longer that these immigrants live in the U.S., the more at-risk they are for diminished health outcomes, given the constant and chronic stressors that they face. Salient stressors faced by undocumented immigrants include socioeconomic disadvantage, harsh living and working conditions, stigmatization and discrimination, high rates of trauma and victimization, constant fear of deportation and family separation, and most importantly limited healthcare access (Garcini et al., 2017; Garcini et al., 2016). Healthcare has been identified as a significant stressor for the majority of the U.S. population (66\%), with immigrants unable to access needed services likely experiencing greater distress than average (American Psychological Association, 2020).

Lack of access to health services is a significant predictor of health risk among undocumented immigrants. Compelling data for this assertion comes from regions that have changed healthcare policy regarding undocumented immigrants and been able to examine population health before and after. Spain, for instance, barred undocumented immigrants from accessing their healthcare system in 2012 and subsequently saw undocumented immigrants less likely to visit their general practitioner and less likely to plan care visits (Jiménez-Rubio \& Vall Castelló, 2020). The exclusion of undocumented immigrants from the health-care system increased the probability of "bad health" reports by $160 \%$ in the undocumented sector of the population (Jiménez-Rubio \& Vall Castelló, 2020). These findings highlight that limited access to healthcare may have profound deleterious effects for undocumented immigrants given that planned healthcare services are needed to maintain good health and sustain functional ability. Likewise, limited healthcare access for undocumented immigrants may have undesirable effects for the host society in the form of higher cost of healthcare for conditions that could have been prevented and/or loss of work.

There are many factors limiting access to healthcare among undocumented immigrants. A primary barrier in the U.S. is their exclusion from federally-subsidized health insurance through the Affordable Care Act (ACA), which forces undocumented immigrants to rely on cash to pay for health services (Patient Protection and Affordable Care Act, 2010). As a result, these immigrants often avoid or delay the use of health services until advanced stages of disease or at a point when their health has significantly impaired their ability to function (Boerner, 2015). In rare instances, some undocumented immigrants have the possibility of receiving healthcare coverage from their employer, but unfortunately these jobs are hard to find and even when insured, undocumented immigrants avoid services due to mistrust and 
fear of deportation (Boerner, 2015). In regions where undocumented individuals are excluded from the healthcare system, fear that interacting with medical professionals might result in immigration enforcement actions is common, and it is often associated with reduced ability to engage in many aspects of healthcare including the use of emergency medical services and acute care, as well as seeking prescription drugs (Berk \& Shur, 2001; Garcini, Cruz et al., 2020; Garcini, Venta et al., 2020). Indeed, fear that seeking healthcare might prompt immigration enforcement action is associated with reduced likelihood of seeking care, including delaying treatment for months for highly infectious diseases and chronic illnesses (Asch et al., 1994; Martinez et al., 2015). Significant barriers of accessibility and availability of appropriate and culturally responsive mental health services exists with immigrant groups making it much more difficult for this population to seek help (Turner et al., 2016). In addition to lacking health insurance and fearing deportation, factors that limit healthcare access among undocumented immigrants include cultural and language barriers, low health literacy, and misinformation, all of which drive existing health disparities as well as morbidity and mortality (Garcini, Cruz et al., 2020; Garcini, Venta et al., 2020; Martinez et al., 2015). Indeed, only $27 \%$ of undocumented immigrants in the U.S. report speaking English "very well," with only $8 \%$ indicating they speak English at home (Migration Policy Institute, 2018). Though the majority of undocumented immigrants in the U.S. are Latinx, originating from Mexico and Central America (68\%) and speak Spanish at home (73\%; Migration Policy Institute, 2018), it is important to note that language and cultural barriers may be even more pronounced for undocumented immigrants who speak languages other than Spanish and, culturally, represent a smaller percentage of the U.S. immigrant population.

Limited access to health services may be particularly distressing for undocumented immigrants in poor health, those who have chronic health conditions, and individuals facing recurring emergency situations (Chavez, 2012). For instance, without primary and preventative care undocumented immigrants with chronic health conditions are unable to access essential resources such as the use of medications, treatment, screenings, or immunizations, that are needed to prevent worsening of health outcomes, diminished quality of life, and impairment of functional ability (Pitkin Derose et al., 2009). Similarly, given that undocumented immigrants often face strenuous work conditions, those in poor health may be more likely to suffer decompensation or acute health crises, which can lead to emergency situations and job loss (Samra et al., 2019). When compared to the U.S. population as a whole, undocumented immigrants have fewer medical visits and rates of hospitalization, yet they are often dependent on the use of emergency services (Berk et al., 2000). Psychologists working in emergency departments and acute inpatient medical units are integral to the health of this at-risk population, as they can facilitate the provision of needed health services and provide referrals for social, counseling, and mental health services that could help immigrants cope with ill health in the face of limited resources and healthcare access. Furthermore, psychologists have the knowledge and tools to recognize and build on the strengths of undocumented immigrants and not only address barriers but also foster liberation and nurture survivance (Comas-Díaz \& Torres Rivera, 2020; Hartmann et al., 2019). 
Over the past decade, there has been increased attention to the potential benefits of an integrated healthcare care model in which psychologists play a vital role collaborating with medical providers and social workers in the provision of services in emergency departments and inpatient medical units (Chenneville \& Gabbidon, 2020). The benefits of having psychologists work as part of the integrated team in inpatient medical settings include increased access to mental health services and referrals, reduced mental health stigma, improved health outcomes associated with insight into psychosocial factors that affect health and illness, better communication between patients and medical providers, and increased sensitivity in addressing the health needs of patients from a cultural and contextual perspective (Chenneville \& Gabbidon, 2020). These benefits can be particularly valuable when working with undocumented immigrants who may not otherwise have access to services that can address the compounded effect that psychosocial stressors may have on their physical health.

For integrated care to be effective, psychologists must be prompt, creative, and flexible in their approach to treatment (Vogel et al., 2012). For instance, treatment sessions in inpatient medical units may be short in duration and limited in number. Successful work with undocumented patients in inpatient medical settings will also depend on the medical team's understanding of the undocumented experience, its legal and social implications, and the ability to prioritize, assess, and address the immigrant's needs from a cultural perspective in a brief amount of time (Herbst et al., 2016). A psychologist that understands the experiences of undocumented immigrants' can connect important patient strengths (e.g., immigrants are luchadores, a word that captures resolve, persistence, and the perseverance needed to not only survive but also thrive) and goals (e.g., a better life, safety for family), with their current health needs and make meaningful plans that integrate traditional and Western methods of healing to promote patients' health without recreating oppressive spaces and procedures or, worse yet, blaming or pathologizing the patient for their condition (Hartmann et al., 2019).

\section{Purpose of Paper}

We illustrate the complex role that psychologists may play in providing integrated health services to undocumented Latinx immigrants in inpatient medical settings through the case study of an undocumented young Latina with acute liver failure. This case highlights the effects of compounded stressors on the physical health of at-risk undocumented immigrants with limited access to healthcare, along with comorbid distress that increases risk for mental illness and diminished health outcomes. We discuss implications and recommendations for the assessment and treatment of undocumented Latinx immigrants in inpatient medical units, as well as discuss needed advocacy efforts to provide additional resources and support to undocumented immigrants facing a health crisis with limited access to medical care.

\section{Case Study}

Ms. A, a 24-year-old undocumented Latina immigrant from Mexico, narrated the following story in Spanish while being a participant in one of our research studies and six months after her sudden visit to an emergency room. Ms. A mentioned that she was referred by her cousin 
to our study, which focused on learning how chronic stress contributes to the development of chronic illnesses among undocumented Latino immigrants. At the time of her participation, Ms. A's physical and mental health had been deteriorating for some time and she had difficulty narrating her story without breaking down in tears. During her interview, Ms. A reported feeling hopeless, helpless and in despair, and mentioned having some intrusive thoughts about death and dying. In compliance with the research protocol, Ms. A was provided with a referral to a low-cost community mental health clinic, along with educational resources in hope to facilitate access to treatment. Unfortunately, as in many of the stories told in our research studies, we do not know what happened to Ms. A or if she ever sought treatment. Nonetheless, it is valuable to consider how her story would have been different if she had a psychologist in her medical team during that initial hospital emergency room visit. Ms. A provided informed consent to use her story for academic purposes. To protect Ms. A, her name has been de-identified and the initial letter to her name has been changed.

Six months prior to her participation in our study, Ms. A presented to an emergency room at a local city hospital, which belonged to the public healthcare system, following an episode of severe abdominal pain and vomiting along with difficulty breathing. Ms. A reported that these symptoms followed months of having migraines, unbearable neck pain, and severe anxiety. Emergency room lab tests showed that Ms. A's symptoms indicated acute liver failure likely caused by an excessive use of over-the-counter pain relievers. Ms. A reported that in order to cope with migraines and chronic neck pain, she had been taking pain relievers (i.e., Tylenol) several times a day in large amounts for over a year as a way to maintain her functional ability. Ms. A did not have medical insurance; thus, she reported avoiding seeking medical help for her migraines and chronic pain. In addition, she denied having a regular healthcare provider stating that she fears discrimination due to her undocumented immigration legal status. At the emergency room, Ms. A was concerned about disclosing personal information for fear that she may be deported or separated from her family. When the doctor informed her that she needed to spend a few days in the hospital, Ms. A became extremely anxious and shared that she would be unable to pay for the hospital stay, any recommended treatments, medications, or follow up visits.

Ms. A immigrated to the U.S. at age 5 with her mother, who is also undocumented. Her father was killed by an organized crime group and they moved to the U.S. in search of safety and security. Three years before the hospital visit, her mother had a stroke that left her unable to work and paralyzed on one side of her body. Consistent with expectations for cisgender women in her cultural context, Ms. A was the primary caretaker to her mother and her two adolescent siblings born in the U.S. For practical reasons she was also the main financial provider for her family. At the time of services, she was working two part-time jobs and had recently quit school due to financial constraints. Ms. A's primary concerns were undocumented status, financial difficulties, and the family's lack of health insurance which made it difficult her family to seek the medical care they needed. For instance, unable to afford consultation and treatment for her migraines and chronic neck pain, Ms. A had been self-medicating and ignoring warning signs, which led to her liver dysfunction. Similarly, medical care for her mother had been neglected due to cost, which resulted in her mother's rapidly deteriorating health. A more affordable alternative for the family has been to pursue 
the services of curanderos or traditional healers as their primary source of care, yet Ms. A reported no reduction in her pain or improvement of her mother's condition. Ms. A hoped that she could find answers to her complex health issues by continuing to use non-traditional sources of healthcare.

Pertaining to mental health, Ms. A told the social worker at the hospital that she felt stressed, but had difficulty verbalizing her symptoms. She mentioned that she avoided thinking about stress because she could not "afford" to have a break down. She mentioned that she was raised to be a strong person and that she needed to regain the fortitude that has always characterized her. Regardless, Ms. A did convey to the social workers that she recently had an "ataque de nervios" [hysterics] during one of her migraine episodes after an argument about finances with her mother. Ataque de nervios is a relevant cultural concept of distress among Latinx immigrants, and especially women (Guarnaccia et al., 2010). Ataque de nervios is considered a "normative expression of acute distress" characterized by "symptoms of intense emotional upset, including acute anxiety, anger, or grief; screaming and shouting uncontrollably; attacks of crying; trembling; heat in the chest rising to the head; becoming verbally and physically aggressive [among other symptoms]" (American Psychiatric Association, 2013, p. 833). A generalized feature of ataque de nervios is a sense of being out of control, and the ataques are often related to a stressful life event, mostly involving the family. Some DSM disorders that have been related to ataque de nervios include panic attacks, Panic Disorder, Conversion Disorder, unspecified or specified forms of Dissociative Disorders, and Intermittent Explosive Disorder (American Psychiatric Association, 2013). Upon disclosure of her ataques, the social worker recommended that Ms. A seek counseling immediately upon discharge in order to address her distress, compounded health issues, and the many contextual stressors that she faced. Ms. A did not have an opportunity to consult with a psychologist during her hospital stay and did not follow up with the recommendation for seeking mental health services upon discharge.

\section{Discussion}

The case presented in this paper represents one of many undocumented Latinx immigrants whose first referral for counseling or mental health services takes place in an emergency room upon experiencing a life-threatening situation. Psychological services in these instances could be extremely valuable, and psychologists working in inpatient medical units could offer at-risk immigrants a plethora of information and valuable resources (Herbst et al., 2016). Psychologists can also provide a conceptualization of the patient that is rooted in an understanding of evidence-based practice in the context of the patients' multiple statuses and identities and how these are relevant to patients' history, presenting concerns, and course of treatment. This intersectional and contextual awareness is consistent with the competencies expected of psychologists (Buchanan \& Wiklund, 2020).

Unfortunately, access to psychological services in inpatient medical units is limited given a shortage of trained bilingual psychologists equipped to meet the complex health needs of undocumented Latinx immigrants. To increase awareness about the valuable role that psychologists can play in medical inpatient setting to address the health needs of undocumented immigrants and to provide an overview of specific contributions that 
psychologists could make as part of the integrated team, we outline practical assessment and treatment roles, along with advocacy recommendations (Table 1).

\section{Establishing Trust or Confianza}

From the initial contact with the patient, a key determinant of effective treatment is the establishment of trust or confianza. When working with undocumented Latinx immigrants, building trust is especially important given that prevalent anti-immigrant rhetoric and actions have increased fear and mistrust among undocumented communities, making it very difficult for these immigrants to open up about their life and struggles (Garcini, Cruz et al., 2020; Garcini, Venta et al., 2020). For example, concerns about the misuse of personal information often increase fear of deportation, family separation, and job loss (Garcini et al., under review). Similarly, fears of discrimination and stigmatization stemming from the portrayal of undocumented immigrants as a burden to the healthcare system and as a public health hazard are likely to influence honest disclosure about existing health challenges and needs that undocumented immigrants may have (Garcini, Cruz et al., 2020; Garcini, Venta et al., 2020). Concerns about victimization related to financial scams or being stuck with large medical bills that the undocumented immigrant will be unable to afford also contribute to mistrust in this immigrant population (Garcini et al., under review).

An essential way in which psychologists can help build trust or confianza between undocumented immigrants and their medical providers is by emphasizing the core principles of Person-Centered Therapy in interpersonal dynamics (Raskin \& Rogers, 2005). Providing undocumented immigrants with respect, empathy, understanding, and acceptance of their cultural and contextual experiences, including past experiences with providers and healthcare systems, is essential to facilitate trust (Raskin et al., 2008). Building personal connections that are based on trust has been identified as an essential component of effective psychotherapy among Latinxs (Bekelman et al., 2019; Falgas-Bague et al., 2019).

Psychologists working from a liberation framework can communicate clearly about health inequities and systemic discrimination and are committed to address them (Comas-Díaz \& Torres Rivera, 2016). For example, psychologists can address one or multiple of the stages of the Healing Ethnocultural and Racial Trauma model (Chavez-Dueñas et al., 2019) including, offering relief, acknowledging ethno-racial trauma, and promoting survivance. In inpatient medical settings, psychologists can work with medical providers and patients to emphasize safeguards to confidentiality and work collaboratively to build safe spaces.

\section{Facilitating Communication and Interactions with Medical Providers}

An important task that psychologists have in inpatient medical settings is to train medical providers to have cultural humility and to be culturally sensitive in their approach and interactions with patients. Cultural humility requires a constant process of self-reflection and willingness to learn from others in a way that fosters honest, respectful, and trustworthy interpersonal interactions (Yeager \& Bauer, 2013). It is important that medical providers stay centered in cultural humility and the patient's lived experiences to avoid harmful overgeneralizations, such as assuming that certain values, behaviors, or life experiences may be held by all Latinxs or by all undocumented immigrants. Similarly, the use of culturally appropriate interpersonal dynamics, assessments, and treatments is essential to facilitate 
understanding of the ways in which culture may relate or contribute to the health of undocumented Latinx immigrants, while also acknowledging that undocumented Latinx immigrants are a heterogeneous group. Acknowledgement of and respect for individual differences aligns with the greater emphasis placed on personalized medicine (Golubnitschaia \& Costigliola, 2010).

Raising awareness about cultural values that may be important among undocumented Latinx immigrants is helpful to facilitate communication and to build safe and respectful environments for patients. For instance, among Latinxs, the value of simpatía refers to striving to maintain harmonious relationships and avoid conflict, which could lead patients to appear agreeable with medical providers even when they may not agree with the provider's recommendations, thereby, increasing risk for miscommunication and possibly less compliance with medical recommendations (Zamudio et al., 2017). Similarly, respeto, which is a measure of power or influence between two people, may lead undocumented Latinx immigrants to offer deference, to act timidly, and to be passive with providers who may be perceived by undocumented immigrants as having a higher status (Zamudio et al., 2017). Often, this can lead to misunderstanding and unanswered questions, which may negatively affect treatment adherence. Attention to modestia or modesty as a relevant value is also important to consider. For instance, among undocumented Latinx immigrants of conservative backgrounds, physical exposure and discussion of sensitive topics (e.g., effect of illness on sexual health) may be challenging and may lead to underreporting, which may be problematic for diagnosis and effective treatment (Juckett, 2013). Another important cultural value among Latinxs is familismo, which emphasizes the need to put one's family above oneself, including protecting family bonds and making contributions to the wellbeing of family members (Campos et al., 2014). Psychologists can be extremely valuable in helping medical providers understand family dynamics and attitudes that may influence treatment and health outcomes for undocumented Latinx immigrants, along with facilitating skills helpful to navigate family issues in a humble, respectful, and culturally sensitive manner (Willerton et al., 2008).

Psychologists can also make meaningful contributions to improve interactions and communication between patients and medical providers by working with undocumented immigrants on the development of basic navigation skills that could lead to more successful encounters and experiences with the healthcare system (Fernandez-Gutierrez, et al., 2018). For instance, this task may include helping patients learn how to ask questions of medical providers and when to ask for clarification of information, developing assertiveness to convey health needs, learning to circumnavigate and problem-solve barriers to treatment, and acquiring skills to locate and evaluate the validity of health information from different sources (e.g., community agencies, social media, online resources, friends/family).

Moreover, by discussing influences that hold sway over their patients' health decisions, such as family, friends, religion, culture, prior medical experiences, or perceived truths regarding health, psychologists can help medical providers better understand immigrants' experiences and different worldviews.

It is not uncommon among undocumented immigrants for misunderstandings regarding the severity of an illness or importance of treatment compliance to stem from limited English 
proficiency, low health literacy, and variations in cultural beliefs about health and illness between the patient and the medical provider (Pitkin Derose et al., 2007). As such, psychologists can devote time to ensure that undocumented immigrants have access to translation services if needed. Furthermore, psychologists can also work with patients to clarify questions and incorrect assumptions and misunderstanding about their illness and its treatment.

\section{Conducting Culture and Context Sensitive Assessments}

Another important step in which psychologist help address the compounded health needs of undocumented Latinx immigrants in inpatient medical units is to facilitate a comprehensive and holistic health assessment. Assessment must be culturally informed and contextually appropriate (American Psychological Association, 2017a). An important aspect to consider is that there are cultural variations in myths and beliefs about the concepts of health and illness, as well as treatment options, which need to be assessed, considered, and discussed with the patient and the medical team in order to facilitate compliance with medical recommendations and to increase treatment effectiveness (Benish et al., 2011; Garcini et al., 2015; McQuaid \& Landier, 2018).

In the case of Ms. A, a psychologist could play a vital role in helping her understand more in-depth her illness by clarifying beliefs about the etiology and prognosis of her health condition, along with identifying her beliefs about the perceived severity and consequences of the disease, which will have treatment implications. Likewise, psychologists can also assist patients in uncovering and discussing personal, external, and cultural factors that may influence their health and illness, such as, unmanaged chronic pain, inadequate use of medication, life stress, inability to afford health services, limited health literacy, and cultural values (Garcini et al., 2015). All of the aforementioned knowledge is essential to increase the patient's knowledge about their health condition, facilitate communication between patients and medical providers, and increase compliance with treatment recommendations. In addition, this information is useful for adequate conceptualization needed to guide treatment and to help motivate behavioral changes necessary to prevent worsening of the illness and to promote good health.

Another key way in which psychologists can assist in the assessment process is in obtaining information to elucidate on the patient's views about treatment and their background in interacting with medical providers and the healthcare system. Assessing the patient's understanding of treatment options such as medication use, check-ups and medical recommendations, and preferences for the use of complementary and alternative medicine can help facilitate treatment effectiveness. Psychologists can also help medical providers learn about patients' experiences and knowledge in navigating healthcare systems, including their ability to communicate effectively with medical providers. In this regard, psychologists can elaborate on patients' perceived trust in providers, fears about disclosing personal information (particularly pertaining to immigration legal status), previous experiences with discrimination or racism, level of acculturation, language barriers, and skills for facilitating communication with medical providers, all of which can influence treatment and compliance with medical recommendations (Garcini et al., 2015). This needs to be done as efficiently as 
possible, which can be facilitated by observing the patient's interactions with different members of the medical team, attending to behavioral cues, and doing a detailed chart review to be used as guide for asking additional questions.

Another important contribution of psychologists pertaining to assessment is facilitating learning about contextual barriers that may interfere with treatment or willingness to engage with healthcare systems among undocumented Latinx immigrants. For instance, to adequately capture the effect of context on the physical and mental health of undocumented immigrants, psychologists can help medical providers understand how socioeconomic adversity (e.g., poverty, hazardous living and working conditions, low education) may interfere with seeking services in order to identify avenues for problem solving (Garcini et al., 2015). As previously mentioned, experiences of discrimination, marginalization, stigmatization, isolation, and/or exploitation that may undermine undocumented immigrants' physical and mental health, as well as how such experiences may influence their interpersonal interactions are also important to consider (Garcini et al., 2015). Additional contextual factors to consider in the assessment process include loss or changes in social support system (e.g., family separation, death of a loved one, disruption in social network), perceived loss of autonomy or freedom due to undocumented legal status, and family environment and dynamics. The undocumented experience often leads to unexpected, rapid and constant changes in support systems, including family dynamics, which increases risk for distress and may undermine immigrants' physical and mental health (Ayon \& Naddy, 2013). Accounting for migration-related trauma, loss and grief associated with the aforementioned factors will also render valuable information to guide treatment recommendations and most importantly avenues to prevent re-traumatization.

Furthermore, psychologists are essential to evaluate patients' mental health from a culturally sensitive perspective. In this regard, psychologists seek to emphasize that in addition to assessing traditional symptom presentation for anxiety, depression, and trauma/stress-related disorders, an assessment of relevant Cultural Concepts of Distress is often needed (previously referred to as Culture-Bound Syndromes; American Psychiatric Association, 2013). Cultural Concepts of Distress refer to "ways that cultural groups experience, understand, and communicate suffering, behavioral problems, or troubling thoughts and emotions" (American Psychiatric Association, 2013, p. 758). The Diagnostic and Statistical Manual (DSM 5th edition) emphasizes three different types of cultural concepts necessary to better understand and document distress among diverse populations (American Psychiatric Association, 2013). These include cultural syndromes, cultural idioms of distress, and cultural explanations or perceived causes. An assessment of somatic symptoms not related to the presenting physical illness and symptoms that may differ from those traditionally reported by the general population (e.g., feeling pins and needles in the hands or feet, feeling heat inside the body, sensations of fluttering in the stomach) can be particularly helpful given that these symptoms may reflect distress from a cultural perspective. Somatic disturbances are important symptoms to assess and document as they relate to Cultural Concepts of Distress rather than to the illness itself. An ability to differentiate somatic symptoms attributable to distress versus the physical illness would help medical providers make more effective recommendations for treatment and would facilitate an understanding of how the body/mind interaction is influencing the health of undocumented immigrants. 
The Cultural Formulation Interview (CFI) included in the Diagnostic and Statistical Manual for Mental Disorders (DSM) $5^{\text {th }}$ is a valuable tool used by psychologists to guide the assessment of relevant Cultural Concepts of Distress (American Psychiatric Association, 2013; Lewis-Fernández et al., 2016).

\section{Treatment Implications and Recommendations}

Using information from the culture and context sensitive assessment described above, psychologists can quickly and more effectively work with undocumented immigrants to assist in the building of empowerment strategies that promote survivance, engage in psychoeducation, and develop a medical safety and emergency plan. As previously mentioned, treatment in medical inpatient settings is often short in duration and limited in number, thus, psychologists and medical providers can more effectively collaborate to prioritize and address needs to empower undocumented immigrants and keep them safe, such as working immediately on the development of a safety and emergency plan.

\section{Empowerment Strategies}

It is common for undocumented immigrants to report feeling helpless or not-in-control of their current situation, particularly in the face of chronic illnesses. Although often attributed to cultural values such as fatalismo; a prevalent belief among Latinx immigrants that emphasizes that fate cannot be changed and that life events are beyond one's control; it is important to consider how helplessness among undocumented immigrants may stem from oppressive factors and limited resources associated with being undocumented (AbraídoLanza et al., 2007). Survivance scholarship points to the importance of eschewing victim narratives that serve to reduce people to their circumstances (Hartmann et al., 2019). Psychologists in medical setting can empower undocumented immigrants to build coping strategies and resources for maintaining wellbeing and prevent the deterioration of their physical health.

The development of coping strategies and stress-management skills are essential to reduce the negative effect that contextual stressors and demanding life and work conditions may have on the health of undocumented immigrants. Psychologists can be essential to give immigrants agency to consider their own preferred coping strategies, along with using a strength-based approach focused on developing and building upon immigrants' dispositional attributes, strengths, qualities, and motivation that contribute to resilience and self-image (Garcini et al., in press; Smith, 2006). For instance, commonly used coping strategies to overcome distress and life adversity among undocumented immigrants include behavioral strategies such as distraction or engaging in pleasurable activities, protecting family unity, reframing adversity, and finding comfort in religion and spirituality (Garcini et al., in press; Kam et al., 2018). Furthermore, psychologists could help to identify effective social support systems that may provide undocumented immigrants with instrumental, informational, and emotional support as needed. Of critical importance is the notion that undocumented immigrants' nurturing their physical and mental health can be a radical act to counteract systemic inequities. Using creativity is necessary given that sources of support may be away from the immigrant (e.g., country of origin) or may change constantly. In many cases, 
psychologists are instrumental in finding immigrant support groups led by community organizations that could be valuable to increase support networks.

Another important task that psychologists may engage in is to assist undocumented immigrants in building skills for a healthy lifestyle within the constraints of physical illness. Indeed, exploring alternatives for healthy living and self-care, such as building strategies to improve sleep, rest, and eating habits, could be particularly helpful and empowering. Identifying risk factors to physical health, such as drinking in the case of Ms. A (given that it may further complicate her liver condition), is another important aspect of self-care that could be emphasized. Additional guidance on the many ways in which psychologists can facilitate the development of empowerment and coping strategies when working with undocumented immigrants is provided in the recently published Guide for Providing Mental Health Services (Cadenas et al., 2020).

\section{Psychoeducation}

Psychoeducation is an extremely important component of working with undocumented immigrants because it facilitates building trust and can influence the effectiveness of treatment. In addition to providing undocumented immigrants with detailed knowledge about the treatment process, including identifying priorities, clarifying expectations, and establishing goals of treatment, psychologists can often work with patients in facilitating an understanding of the relationship between their physical and mental health from a contextually and culturally sensitive framework (Robiner et al., 2014). The mind and body are interconnected and inseparable; in order for an individual to achieve wellness, their mental and physical health must be addressed conjointly (Littrell, 2008). Unfortunately, mental health stigma is widespread among undocumented communities, which makes it difficult for immigrants to identify and accept how contextual stress and life adversity may be impacting their health, including the worsening of physical illnesses (Saechao et al., 2012). In the case of Ms. A, ensuring that she understands how life stress may have contributed to her chronic pain and self-medication, which in turn led to liver failure and its associated negative consequences, is necessary to facilitate changes needed in Ms. A's contextual environment. Facilitating an understanding of the mind-body connection, will also increase motivation to engage in evidence-based strategies to reduce stress associated with the contextual environment.

As emphasized above under assessment implications, any discussion of mental health distress with undocumented immigrants must consider relevant cultural concepts of distress prevalent in their communities. This approach requires that psychologists and medical providers develop culturally competent knowledge, and, if possible, seek consultation with experts. To provide the most effective mental health interventions to immigrant groups, several guiding principles have been proposed by La Roche et al. (2017) and Casas (2017). One important movement in mental health treatment has been cultural evidenced-based psychotherapies (CEMPs). CEBPs refer to evidenced based interventions that emphasize not only race and ethnicity, but also integrate culturally proximal variables, such as, socioeconomic status, environmental challenges, healthcare access, and religion in order to understand their compounded effect on health (La Roche, 2017). By integrating cultural and 
proximal factors into treatment, psychologists can work with medical providers to develop a better understanding of the barriers and facilitators that may influence health, treatment, and compliance with medical recommendations among immigrant patients, and at the same time, build a safe environment grounded on respect, acceptance and mutual understanding of immigrants' complex lives and compounded health challenges.

\section{Development of a Medical Safety and Emergency Plan}

Given the limited access to healthcare faced by undocumented immigrants, along with many competing needs and social and economic barriers that may interfere with treatment, an essential step in working with undocumented immigrants in inpatient medical settings is the development of a medical safety and emergency plan (Rath, 2013). The initial phase in the development of this plan involves working with undocumented immigrants to facilitate an understanding of their illness, its implications, and the importance of compliance with medical recommendations. For instance, in the case of Ms. A ensuring that she understands the severity and potentially life threatening consequences of liver failure should treatment be neglected, along with facilitating an understanding of factors that may worsen her condition (e.g., self-medication, stress), is essential to increase insight about the need for compliance and agreement with a medical treatment plan. Psychologists can make use of motivational interviewing and value-based adaptations in working with patients to help them prioritize their medical treatment in the face of other pressing needs (e.g., financial, family responsibilities; Añez et al., 2008; Lewis-Fernandez \& Diaz, 2002; Oh \& Lee, 2016). Motivational interviewing has been found to be useful in clinical medical settings, and even one session has been shown to be effective in motivating action towards achieving healthrelated goals (VanBuskirk \& Wetherell, 2014). Among Latinos, cultural and value-based adaptations to motivational interviewing have been shown to be effective in improving a wide range of health outcomes, including adherence to medical recommendations (Bahafzallah et al., 2020; Palacio et al., 2015; Rocha-Goldberg et al., 2010).

In working with undocumented immigrants, psychologists can assist in the development of two types of medical emergency plans. On one hand, a plan to comply with medical recommendations in order to keep in good health and/or prevent health deterioration; on the other a plan to strategize in case of subsequent medical emergencies. For instance, in the case of the first plan, psychologists can work with patients to develop strategies for facilitating access to the purchasing of needed medications at low-cost, identify access to a regular source of care with a preferred community clinic or safety-net provider, and plan for periodic check-ups. This could also include addressing and incorporating alternative medicine practices into the treatment plan. To increase compliance with medical recommendations, psychologists can also work with undocumented immigrants in brainstorming and exploring ways that can make best use of family or community resources and support, such as enrolling the support of loved ones or learning to navigate informal community-embedded support practices already taking place.

Pertaining to emergency planning, psychologists can assist undocumented immigrants in learning to identify and distinguish chronic versus acute symptoms, as well as emergency symptoms that may signal a need for seeking urgent or emergency care. Fear of deportation 
and/or inability to afford services lead many undocumented immigrants to ignore their symptoms, including those that may be life threatening, as to avoid the use of health services and potentially negative consequences (e.g., high cost, misuse of information that may lead to deportation). Psychologists could assist immigrants in gaining insight about their symptoms, including writing a list of symptoms to be monitored, which could be helpful to guide decision making and determine the type of medical care needed at a given time. When appropriate and warranted, others may also participate in this process to support the undocumented immigrant patient's health. These collective approaches are consistent with liberatory practices (Chavez-Dueñas et al., 2019; Comas-Díaz \& Torres Rivera, 2020).

Another important component of building a safety and an emergency plan in inpatient medical units is to assist undocumented immigrants in identifying options for accessible health services and treatments, along with ensuring that immigrants know their legal rights (Pitkin Derose et al., 2007). Although these are tasks often undertaken by social workers, psychologists can help facilitate the aforementioned type of communication and augment the work by helping build self-monitoring and or behavioral plans to ensure plans have the highest likelihood of being carried out. For instance, psychologist can collaborate with social workers in identifying low-cost, convenient and accessible routine and emergency health services for undocumented immigrants, including locating facilities that may have bilingual staff or translators available at low cost. This aspect of planning also includes writing down important phone numbers and addresses of potential medical providers, community clinics and hospitals, their hours of operation, policies and procedures, and having a rough estimate for service costs. Many community clinics and hospitals have a list of frequently asked questions that could provide valuable information for undocumented immigrants having difficulty accessing and affording health services. Moreover, in order to make treatment accessible, psychologists can also work with undocumented immigrants on making a budget and a plan for saving money that could be used for health services and treatment. It is equally important to assist undocumented immigrants in identifying sources that can inform them about their legal rights in cases of medical emergencies given that policies and regulations vary across states (Pitkin Derose et al., 2007).

Finally, another important component of building a safety and emergency plan is to assist undocumented immigrants in strategizing about how to address other relevant responsibilities while sick. This planning could be particularly helpful in reducing distress and preventing the patient from neglecting their health in the face of other competing needs (Cervantes et al., 2017) including pointing to important sources of community support. For instance, in the case of Ms. A assisting her in brainstorming options to address the caregiving needs of her ill mother and her two younger siblings, along with developing a plan to properly and safely notify her job when unable to work, could be useful although challenging. Psychologists can encourage undocumented immigrants to discuss the aforementioned medical safety and emergency plan with family and/or friends in order to build support and make the plan work more effectively in case of need. Scheduling periodic rehearsals of the medical emergency plan in collaboration with the patient's family or support system would also be helpful, including what steps need to be taken first and who would be in charge of each step-in order to implement the plan efficiently. This kind of check-in is not only helpful in building skills but consistent with cultural values for 
relationship building (e.g., personalismo) that engender trust between providers and patients and consistent with liberatory practices that build relationships of mutual trust and aid (Chavez-Dueñas et al., 2019; Comas-Díaz \& Torres Rivera, 2020).

\section{Advocacy Implications and Recommendations}

Embedded as ethical principles in the practicing of psychology and counseling are the need for responsibility, justice, and the respect for people's rights and dignity, especially for those that are vulnerable (ACA, 2014; APA, 2017b). Accordingly, this principle entails that professionals working with vulnerable populations, such as undocumented immigrants facing challenging health issues with limited resources and access to healthcare, become involved in advocacy efforts to make access to quality medical services a possibility. Working with undocumented immigrants, psychologists can ensure that these patients' humanity is named, valued, and cared for with the same vigor as any other patient. These efforts could focus, for example, on identifying ways to facilitate referrals to mental health and medical providers serving immigrant communities or working together in the development of educational resources or guides that can inform plans for action when undocumented immigrants present with comorbid physical and mental health conditions. The provision of interdisciplinary services, at low-cost, that could be tailored to meet the complex health needs of undocumented immigrants in inpatient medical settings must be prioritized, including the expansion of safety-net hospitals and the provision of specialized services. Connecting patients with advocacy organizations that address these health inequities can aid in their own liberation (Chavez-Dueñas et al., 2019). This also includes building infrastructure such as websites to research drug costs or search engines that can facilitate finding medical and mental health providers at convenient locations, bilingual health services, and referrals to reliable legal counsel for assisting those in complex situations (e.g., in need of recurrent treatment or hospice care).

Advocacy on behalf of undocumented immigrants with complex health needs is also needed to build a competent workforce. Specifically, advocacy should highlight the need for fostering cultural humility and the development, dissemination, and sustainability of training efforts for working with at-risk immigrants using culturally and contextually sensitive frameworks, conceptualizations, and tools (APA Presidential Task Force on Immigration, 2013). Currently training efforts aimed at fostering cultural humility and competence within medical settings are limited, which increases risk for further harm. Advocating for training in the development of specific skills - such as self-reflection, building trust and creating safe spaces, addressing cultural myths and beliefs about health and illness in a respectful manner, understanding the role of alternative medicine and traditional healing practices, assessment considerations, and learning how cultural factors and interpersonal dynamics may influence treatment and interactions with health providers, is needed to provide interdisciplinary quality care to undocumented patients.

Another important focus of advocacy efforts is on the development, dissemination, and sustainability of training aimed at addressing and minimizing the effect of implicit biases on the provision of services to undocumented immigrants. Implicit biases have detrimental effects on patient-provider interactions, treatment decisions, treatment adherence, and 
patient health outcomes (Hall et al., 2015). Biased information about who undocumented immigrants are and a poor understanding of the immigration experience and the complex and compounded challenges that undocumented immigrants face, have propagated negative stereotypes about this immigrant community that are associated with negative health and social consequences (Garcini et al., 2020). Advocacy to support research efforts that can evaluate the effectiveness of training efforts aimed at targeting implicit biases and their effect on patient-provider interactions are essential to prevent further harm and protect the health of this marginalized population.

\section{Conclusion}

Undocumented immigrants form a significant portion of underserved individuals in the U.S. and are at increased physical and mental health risk due to the challenging life and working conditions that they face, including limited access to healthcare. Chronic stress from life circumstances is often a catalyst for poor health, which for the uninsured and underserved leads to a cycle that results in diminished well-being, quality of life, and, in most severe cases, the loss of life. Increasing access to interdisciplinary, affordable, safe, and culturally competent inpatient medical care for this vulnerable population is necessary to reduce risk and prevent further harm. Constantly changing immigration policies have impeded efforts for undocumented immigrants to receive adequate healthcare. Continuous advocacy efforts, including advocating for building a competent workforce and fostering interdisciplinary collaboration between medical and mental health providers, are needed to ensure social justice and the protection of human rights of vulnerable immigrant populations.

\section{Acknowledgments}

Funding: National Institutes of Health, National Heart, Lung, and Blood Institute (NHLBI) (K01HL150247; PI: Garcini).

\section{References}

Abraído-Lanza AE, Viladrich A, Flórez KR, Céspedes A, Aguirre AN, \& De La Cruz AA (2007). Commentary: Fatalismo reconsidered: A cautionary note for health-related research and practice with Latino populations. Ethnicity \& Disease, 17(1), 153-158.

American Counseling Association. (2014). 2014 ACA code of ethics. Retrieved from https:// www.counseling.org/resources/aca-code-of-ethics.pdf

American Psychiatric Association. (2013). Diagnostic and statistical manual of mental disorders (5th ed.). American Psychiatric Publishing.

American Psychological Association. (2017a). Multicultural guidelines: An ecological approach to context, identity, and intersectionality. Retrieved from: http://www.apa.org/about/policy/ multicultural-guidelines.pdf

American Psychological Association. (2017b). Ethical principles of psychologists and code of conduct (2002, amended effective June 1, 2010, and January 1, 2017). https://www.apa.org/ethics/code/

American Psychological Association. (2020). Stress in America 2020: A national mental health crisis. https://www-apa-org/news/press/releases/stress/2020/report-october

American Psychological Association Presidential Task Force on Immigration (2013). Crossroads: The psychology of immigration in the new century. Retrieved from http:/www.apa.org/topics/ immigration/executive-summary.pdf 
Añez LM, Silva MA, Paris M Jr., \& Bedregal LE (2008). Engaging Latinos through the integration of cultural values and motivational interviewing principles. Professional Psychology: Research and Practice, 39(2), 153-159.

Asch S, Leake B, \& Gelberg L (1994). Does fear of immigration authorities deter tuberculosis patients from seeking care? Western Journal of Medicine, 161(4), 373-376.

Ayón C \& Naddy MBG (2013). Latino immigrant families' social support networks: Strengths and limitations during a time of stringent immigration legislation and economic insecurity. Journal of Community Psychology, 41, 359-377.

Bahafzallah L, Hayden KA, Raffin Bouchal S, Singh P, \& King-Shier KM (2020). Motivational interviewing in ethnic populations. Journal of Immigrant and Minority Health, 22(4), 816-851. https://doi.org/10.1007/s10903-019-00940-3

Bekelman DB, Fink RM, Sannes T, Kline DM, Borrayo EA, Turvey C, \& Fischer SM (2020). Puente para cuidar (bridge to caring): A palliative care patient navigator and counseling intervention to improve distress in Latino/as with advanced cancer. Psycho-Oncology, 29(4), 688-695. https:// doi.org/10.1002/pon.5313

Benish SG, Quintana S, \& Wampold BE (2011). Culturally adapted psychotherapy and the legitimacy of myth: A direct-comparison meta-analysis. Journal of Counseling Psychology, 58(3), 279-289. https://doi.org/10.1037/a0023626

Berk ML, Schur CL, Chavez LR, \& Frankel M (2000). Healthcare use among undocumented Latino immigrants. Health Affairs, 19(4), 51-64.

Berk ML, \& Schur CL (2001). The effect of fear on access to care among undocumented Latino immigrants. Journal of Immigrant Health, 3(3), 151-156.

Boerner H (2015). Migrating care: How the ACA does and does not address undocumented immigration. Physician Leadership Journal, 2(2), 44-46.

Buchanan NT, \& Wiklund LO (2020). Why clinical science must change or die: Integrating intersectionality and social justice. Women \& Therapy, 43(3-4), 309-329. https://doi.org/ 10.1080/02703149.2020.1729470

Cadenas GA, Campos L, Minero LP, \& Aguilar C (2020, April 20). A guide for providing mental health services to immigrants impacted by changes to DACA and the COVID-19 pandemic. Retrieved from: https://www.informedimmigrant.com/guides/daca-mental-health-providers/

Campos B, Ullman JB, Aguilera A, \& Dunkel Schetter C (2014). Familism and psychological health: The intervening role of closeness and social support. Cultural Diversity and Ethnic Minority Psychology, 20(2), 191-201. https://doi.org/10.1037/a0034094

Casas MJ (2017) Caution: Immigration maybe harmful to your mental health. In Casas JM, Suzuki LA, Alexander CM, \& Jackson MA (Eds.), Handbook of Multicultural Counseling. Sage Publications.

Cervantes L, Fischer S, Berlinger N, Zabalaga M, Camacho C, Linas S, \& Ortega D (2017). The illness experience of undocumented immigrants with end-stage renal disease. JAMA Internal Medicine, 177(4), 529-535. https://doi.org/10.1001/jamainternmed.2016.8865

Chavez LR (2012). Undocumented immigrants and their use of medical services in Orange County, California. Social Science \& Medicine, 74(6):887-893. https://doi.org/10.1016/ j.socscimed.2011.05.023

Chavez-Dueñas NY, Adames HY, Perez-Chavez JG, \& Salas SP (2019). Healing ethno-racial trauma in Latinx immigrant communities: Cultivating hope, resistance, and action. American Psychologist, 74(1), 49-62. https://doi.org/10.1037/amp0000289

Chenneville T, \& Gabbidon K (2020) Application of the APA ethics code for psychologists working in integrated care settings: Potential conflicts and resolutions. Ethics \& Behavior, 30(4), 264-274. https://doi.org/10.1080/10508422.2019.1683739

Comas-Díaz L, \& Torres Rivera E (Eds.) (2020). Liberation Psychology: Theory, method, practice, and social justice. American Psychological Association.

Falgas-Bague I, Wang Y, Banerjee S, Ali N, DiMarzio K, Palao Vidal D, \& Alegría M (2019). Predictors of adherence to treatment in behavioral health therapy for Latino immigrants: The importance of trust. Frontiers in Psychiatry, 10, 817. https://doi.org/10.3389/fpsyt.2019.00817 
Fazel-Zarandi MM, Feinstein JS, \& Kaplan EH (2018). The number of undocumented immigrants in the United States: Estimates based on demographic modeling with data from 1990 to 2016. PLOS ONE, 13(9), e0201193. https://doi.org/10.1371/journal.pone.0201193

Fernández-Gutiérrez M, Bas-Sarmiento P, Albar-Marín MJ, Paloma-Castro O \& Romero-Sánchez $\mathrm{JM}(2018)$ Health literacy interventions for immigrant populations: a systematic review. International Nursing Review 65, 54-64.

Garcini LM, Cruz P, \& Abraham C (2020, October 18). US elections 2020: Undocumented status and mental healthcare access. Medical News Today. Retrieved from Https:// www.medicalnewstoday.com/articles/us-election-2020-undocumented-status-and-mentalhealthcare-access.

Garcini LM, Domenech Rodríguez MM, Mercado A, \& Paris M (2020). A tale of two crises: The compounded effect of COVID-19 and anti-immigration policy in the United States. Psychological Trauma: Theory, Research, Practice, and Policy, 12(S1), S230-S232. https://doi.org/10.1037/ tra0000775

Garcini LM, Murray K, Barnack-Tavlaris J, \& Klonoff E (2015). Cultural concerns. In Andrasik F, Goodie J, \& Peterson A (Eds.), Biopsychosocial assessment in clinical health psychology: A handbook (pp. 369-389). Guildford Press

Garcini LM, Murray K, Zhoe A, Klonoff EA, Myers M, \& Elder JP (2016). Mental health of undocumented immigrant adults in the United States: A systematic review of methodology and findings. Journal of Immigrant and Refugee Studies, 14: 1-25. https://doi.org/ 10.1080/15562948.2014.998849

Garcini LM, Renzaho AMN, Molina M, \& Ayala GX (2017). Health related quality of life among Mexican-origin Latinos varying in immigration legal status. Ethnicity \& Health, 1, 1-16. https:// doi.org/10.1080/13557858.2017.1283392

Garcini LM, Rosenfeld J, Kneese G, Bondurat R, \& Kanzler K (under review). Dealing with distress from the COVID-19 Pandemic: Mental health stressors and coping strategies in vulnerable Latinx communities. Health and Social Care in the Community.

Garcini LM, Venta A, \& Abraham C (2020, December 3). US elections result: Impact on healthcare for undocumented communities. Medical News Today. Retrieved from https:// www.medicalnewstoday.com/articles/us-election-result-impact-on-healthcare-for-undocumentedcommunities

Golubnitschaia O, \& Costigliola V (2010). Common origin but individual outcomes: time for new guidelines in personalized healthcare. Personalized Medicine, 7(5), 561-568.

Guarnaccia PJ, Lewis-Fernandez R, Pincay IM, Shrout P, Guo J, Torres M, Canino G, \& Alegria M (2010). Ataque de nervios as a marker of social and psychiatric vulnerability: Results from the NLAAS. The International Journal of Social Psychiatry, 56(3), 298-309. https://doi.org/ $10.1177 / 0020764008101636$

Hall WJ, Chapman MV, Lee KM, Merino YM, Thomas TW, Payne BK, Eng E, Day SH, \& CoyneBeasley T (2015). Implicit racial/ethnic bias among health care professionals and its influence on health care outcomes: A systematic review. American Journal of Public Health, 105(12), e60-e76. https://doi.org/10.2105/AJPH.2015.302903

Hartmann WE, Wendt DC, Burrage RL, Pomerville A, \& Gone JP (2019). American Indian historical trauma: Anticolonial prescriptions for healing, resilience, and survivance. American Psychologist, 74(1), 6-19. https://doi.org/10.1037/amp0000326

Herbst RB, Bernal DR, Terry J, \& Lewis B (2016). Undocumented Latina/o immigrants in multidisciplinary settings: Behavioral health providers' role in promoting optimal, ethical healthcare. Journal for Social Action in Counseling \& Psychology, 8(1), 89-108. https://doi.org/ 10.33043/JSACP.8.1.89-108

Jiménez-Rubio D, \& Vall Castelló J (2020). Limiting health-care access to undocumented immigrants: A wise option? Health Economics, 29(8), 878-890. https://doi.org/10.1002/hec.4115

Juckett G (2013). Caring for Latino patients. American Family Physician, 87(1), 48-54.

Kam JA, Perez Torres D, \& Steuber Fazio K (2018). Identifying individual- and family-level coping strategies as sources of resilience and thriving for undocumented youth of Mexican origin. Journal of Applied Communication Research, 46(5), 641-644. 
La Roche M, Christopher MS, \& West LM (2017). Toward a cultural evidenced-based psychotherapy. In Casas JM, Suzuki LA, Alexander CM, \& Jackson MA (Eds.), Handbook of Multicultural Counseling. Sage Publications.

Lewis-Fernández R, \& Díaz N (2002). The cultural formulation: A method for assessing cultural factors affecting the clinical encounter. Psychiatry Quarterly, 73(4):271-95. https://doi.org/ 10.1023/a:1020412000183

Lewis Fernández R, Aggarwal NK, Hinton L, Hinton DE, \& Kirmayer LJ (Eds.) (2016). DSM-5: Handbook on the Cultural Formulation Interview. American Psychiatric Press.

Littrell J (2008). The mind-body connection. Social Work in Health Care, 46(4), 17-37. https://doi.org/ 10.1300/J010v46n04_02

Martinez O, Wu E, Sandfort T, Dodge B, Carballo-Dieguez A, Pinto R, Rhodes SD, Moya E, \& Chavez-Baray S (2015). Evaluating the impact of immigration policies on health status among undocumented immigrants: A systematic review. Journal of Immigrant and Minority Health, 17(3), 947-970. https://doi.org/10.1007/s10903-013-9968-4

McQuaid EL, \& Landier W (2018). Cultural issues in medication adherence: Disparities and directions. Journal of General Internal Medicine, 33(2), 200-206. https://doi.org/10.1007/ s11606-017-4199-3

Migration Policy Institute. (2018). Profile of the unauthorized population: United States. Retrieved April 4, 2021 from https://www.migrationpolicy.org/data/unauthorized-immigrant-population/ state/US.

Oh H, \& Lee C (2016). Culture and motivational interviewing. Patient Education and Counseling, 99(11), 1914-1919. https://doi.org/10.1016/j.pec.2016.06.010

Pitkin Derose K, Bahney BW, Lurie N, \& Escarce JJ (2009). Review: Immigrants and health care access, quality, and cost. Medical Care Research and Review, 66(4), 355-408. https://doi.org/ $10.1177 / 1077558708330425$

Palacio AM, Uribe C, Hazel-Fernandez L, Li H, Tamariz LJ, Garay SD, \& Carrasquillo O (2015). Can phone-based motivational interviewing improve medication adherence to antiplatelet medications after a coronary stent among racial minorities? A randomized trial. Journal of General Internal Medicine, 30(4), 469-475. https://doi.org/10.1007/s11606-014-3139-8

Passel JS, Cohn D, Krogstad JM, \& Gonzalez-Barrera A (2014). As growth stalls, unauthorized immigrant population becomes more settled. Pew Research Center. https://www.pewresearch.org/ hispanic/2014/09/03/as-growth-stalls-unauthorized-immigrant-population-becomes-more-settled/

Patient Protection and Affordable Care Act (PPACA), Pub. L. No. 111-148, 124 Stat. 119 (March 23, 2010).

Pitkin Derose K, Escarce JJ, \& Lurie N (2007). Immigrants and health care: Sources of Vulnerability. Health Affairs, 26(5), 1258-1268.

Raskin NJ, \& Rogers CR (2005). Person-centered therapy. In Corsini RJ \& Wedding D (Eds.), Current psychotherapies(p. 130-165). Thomson Brooks/Cole Publishing Co.

Raskin NJ, Rogers CR, \& Witty MC (2008). Client-centered therapy. In Wedding D \& Corsini RJ (Eds.), Current Psychotherapies (pp. 141-186). Thomson Higher Education.

Rath C (2013), Undocumented Patients and the Not-So-Safe Safety Net. Hastings Center Report, 43: inside back cover-inside back cover. https://doi.org/10.1002/hast.212

Robiner WN, Dixon KE, Miner JL, \& Hong BA (2014). Psychologists in medical schools and academic medical centers: Over 100 years of growth, influence, and partnership. American Psychologist, 69(3), 230-248. https://doi.org/10.1037/a0035472

Rocha-Goldberg M del P, Corsino L, Batch B, Voils CI, Thorpe CT, Bosworth HB, \& Svetkey LP (2010). Hypertension Improvement Project (HIP) Latino: Results of a pilot study of lifestyle intervention for lowering blood pressure in Latino adults. Ethnicity \& Health, 15(3), 269-282. https://doi.org/10.1080/13557851003674997

Saechao F, Sharrock S, Reicherter D, Livingston JD, Aylward A, Whisnant J, Koopman C, \& Kohli S (2012). Stressors and barriers to using mental health services among diverse groups of firstgeneration immigrants to the United States. Community Mental Health Journal, 48(1), 98-106. https://doi.org/10.1007/s10597-011-9419-4 
Samra S, Taira BR, Pinheiro E, Trotzky-Sirr R, \& Schneberk T (2019). Undocumented patients in the emergency department: Challenges and opportunities. The Western Journal of Emergency Medicine, 20(5), 791-798.

Smith EJ (2006). The strength-based counseling model. The Counseling Psychologist, 34(1), 13-79. https://doi.org/10.1177/0011000005277018

Turner EA, Cheng HS, Llamas J, Tran A, Hill K, Loung-Tran C, Fretts JM, \& Mercado A (2016). Factors Impacting The Current Trends in the Use of Outpatient Psychiatric Treatment Among Diverse Ethnic Groups. Current Psychiatric Reviews, 12, 199-220.

VanBuskirk KA, \& Wetherell JL (2014). Motivational interviewing with primary care populations: A systematic review and meta-analysis. Journal of Behavioral Medicine, 37(4), 768-780. https:// doi.org/10.1007/s10865-013-9527-4

Vogel ME, Kirkpatrick HA, Collings AS, Cederna-Meko CL, \& Grey MJ (2012). Integrated care: Maturing the relationship between psychology and primary care. Professional Psychology: Research and Practice, 43(4), 271-280. https://doi.org/10.1037/a0029204

Willerton E, Dankoski ME, \& Martir JFS (2008). Medical family therapy: A model for addressing mental health disparities among Latinos. Families, Systems, \& Health, 26(2), 196-206. https:// doi.org/10.1037/1091-7527.26.2.196

Yeager KA, \& Bauer-Wu S (2013). Cultural humility: essential foundation for clinical researchers. Applied Nursing Research, 26(4), 251-6. https://doi:10.1016/j.apnr.2013.06.008.

Zamudio CD, Sanchez G, Altschuler A, \& Grant RW (2017). Influence of language and culture in the primary care of Spanish-speaking Latino adults with poorly controlled diabetes: A qualitative study. Ethnicity \& Disease, 27(4), 379-386. https://doi.org/10.18865/ed.27.4.379 


\section{Clinical Impact Statement}

\section{Question:}

We ask how can psychologists work as members of interdisciplinary teams in medical inpatient settings to address the complex health needs of undocumented Latinx immigrants.

\section{Findings:}

Through a case study, we illustrate practical assessment and intervention implications and recommendations for working with undocumented Latinx immigrants in inpatient medical settings, along with a discussion of needed advocacy efforts.

\section{Meaning:}

Psychologist have a key role in conducting culture and context sensitive assessments and treatments for undocumented immigrants in inpatient medical settings, as well as facilitating communication with the medical team, which is essential to inform treatment effectiveness.

\section{Next Steps:}

Research efforts are needed to further document the impact that psychologists can have on improving patient-provider interactions and health outcomes for undocumented immigrants in inpatient medical settings. 


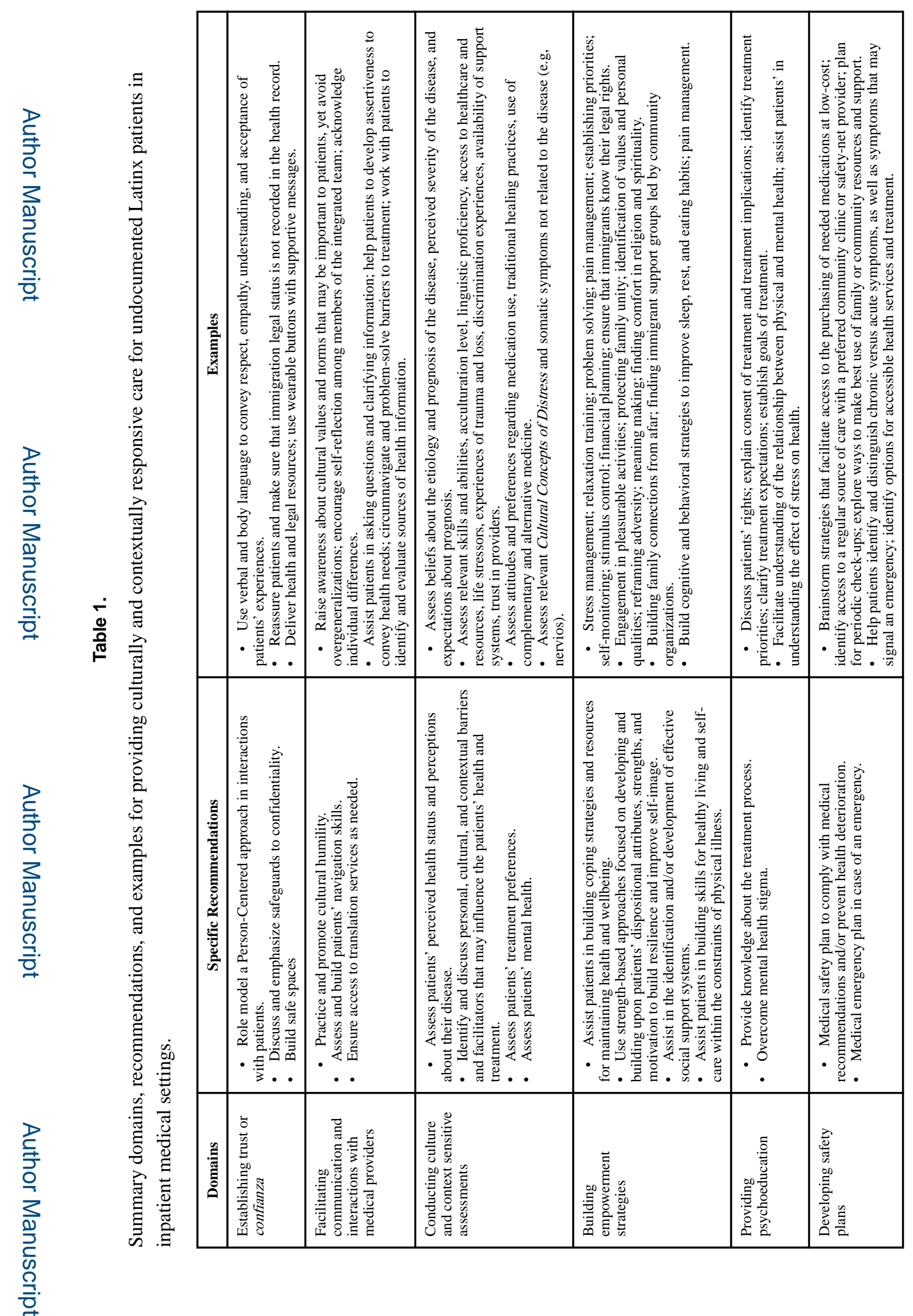

Psychotherapy (Chic). Author manuscript. 